\title{
Análisis del impacto presupuestal de mometasonal formoterol en enfermedad pulmonar obstructiva crónica en Colombia
}

\author{
Analysis of the economic impact of mometasone/formoterol \\ in chronic obstructive pulmonary disease in Colombia
}

\author{
Jacqueline Pavia, MD.(1), Elkin V. Lemos, MD., PhD.(1); Margarita Restrepo, MD. MSc. ${ }^{(1)}$; \\ FIDEL LOZANO, MD. ${ }^{(1)}$
}

\section{Resumen}

INTRODUCCIÓN: la mometasona/formoterol es un inhalador presurizado de dosis medida que contiene corticosteroides y un agonista beta de acción prolongada que proporciona un control eficiente de los síntomas en los casos de EPOC. La dosis recomendada es de dos inhalaciones, dos veces al día.

OBJETIVO: desarrollar un análisis del impacto presupuestal entre 2013 y 2017 con la introducción de mometasona/formoterol en el sistema de salud de Colombia. Este análisis estima las implicaciones financieras del uso de este medicamento en pacientes mayores de 40 años con enfermedad pulmonar obstructiva crónica.

MATERIAL Y MÉTODO: mediante la perspectiva del sistema de seguridad social se diseñó un modelo de impacto presupuestal para determinar el efecto de la introducción de mometasona/ formoterol sobre los recursos del sistema de salud. Se siguieron las directrices y recomendaciones aplicadas para estos estudios, teniendo en cuenta la población objetivo específico, la perspectiva del estudio y el horizonte temporal. La información clínica y epidemiológica de la enfermedad se obtuvo de la literatura médica disponible (guías colombianas) y los costos se tomaron del manual nacional SISMED (sistema de información de precios de medicamentos) para el año 2013. El análisis de costos se realizó en dos escenarios: con y sin descuento, usando una tasa constante del 3\% para los años estudiados. La robustez de los resultados se evaluó mediante un análisis sensibilidad univariado.

RESULTADOS: por medio del modelo basado en la población para una cohorte de 46.927 .125 personas en Colombia y aplicando los datos epidemiológicos locales, se estima que un total de 119.962 pacientes con enfermedad pulmonar obstructiva crónica tendrían la indicación específica para recibir tratamiento con una combinación fija. Los costos netos anuales (2013-2017) con la inclusión de mometasona/formoterol fueron $\$ 88.050$; $\$ 88.052$; $\$ 88.053$; $\$ 88.053$ y $\$ 88.054$ millones de pesos colombianos respectivamente para cada año. El impacto del presupuesto total de la utilización de mometasona/formoterol (sin descuento) en un período de tiempo de 5 años es de $\$ 11.7$ millones de pesos colombianos (11 millones aproximados). El análisis de sensibilidad se enfocó sobre el impacto total en el presupuesto y su relación con la adherencia de las combinaciones de dosis fijas

CONCLUSIÓN: los resultados del análisis del impacto del presupuesto evidencian que la inclusión de la mometasona/formoterol en el tratamiento de la enfermedad pulmonar obstructiva crónica en comparación con otras combinaciones de dosis fijas, tiene el potencial de reducir los costos totales del presupuesto en el sistema de salud colombiano.

Palabras clave: enfermedad pulmonar obstructiva crónica, impacto presupuestal, mometasona.

\footnotetext{
Abstract

INTRODUCTION: mometasone/formoterol is a metered-dose pressurized inhaler that contains corticosteroids and a long-acting beta-agonist; it provides efficient symptom control in cases of COPD. The recommended dose is two inhalations, twice daily.
}

\footnotetext{
${ }^{(1)}$ Medical Affairs, MSD Colombia. Bogotá Colombia.

Correspondencia: Jacqueline Pavia, Calle 100 No. 7-33 piso 6, Bogotá Colombia. Correo electrónico: jacqueline.pavia.albor@ merck.com

Recibido: 21/01/14, Aprobado 20/02/14.
} 
OBJECTIVE: to develop an analysis of the economic impact of the introduction of mometasone/formoterol in the Colombian healthcare system over the period from 2013 to 2017 . This analysis gives an estimate of the financial implications of the use of this medication in patients older than 40 years with chronic obstructive pulmonary disease.

MATERIALS AND METHODS: from the perspective of the healthcare system, a model was designed for determining the effect of the introduction of mometasone/formoterol on the system's resources. The guidelines and recommendations used in such studies were followed, taking into account the specific target population, the study's perspective, and the time frame. Clinical and epidemiological information was drawn from the available medical literature (Colombian guidelines), and costs were gathered from the national handbook of drug prices (SISMED) for the year 2013. Cost analysis was done in two scenarios: with and without discount, using a constant 3\%-rate for the years under study. The robustness of the results was evaluated by a univariate sensitivity analysis.

RESULTS: through the population-based model, for a cohort of 46.927 .125 people in Colombia, and applying epidemiological data, it is estimated that a total number of 119.962 patients would have the specific indication to receive treatment with a fixed combination. The net annual costs (2013-2017) with the inclusion of mometasone-formoterol were \$88.050; \$88.052; \$88.053; \$88.053; and \$88.054 Colombian pesos respectively for each year. The impact of the total budget of mometasone/formoterol (without discount) over a five-year period is $\$ 11.76$ (11 million Colombian pesos, approximately). The sensitivity analysis was focused on the total impact on the budget and its relation with the compliance to fixed-dose combinations.

CONCLUSION: the results of the analysis of budget impact shows that the inclusion of mometasone/formoterol in the treatment of chronic obstructive pulmonary disease, compared with other fixed-dose combinations, has the potential to reduce the total costs of the budget in the Colombian healthcare system.

Keywords: chronic obstructive pulmonary disease, economic impact, mometasone.

\section{Introducción}

La enfermedad pulmonar obstructiva crónica (EPOC), es una enfermedad crónica con impacto en la salud pública, que ha aumentado de manera acelerada tanto en Colombia como en el mundo $(1,2)$.

Es una de las enfermedades más frecuentes en la población mayor de 40 años. Su prevalencia aumenta a medida que avanza la edad y es mayor en los países con un consumo alto de cigarrillo. Es más común en hombres, que tradicionalmente han fumado más, que en mujeres, aunque en éstas, la prevalencia aumenta a medida que se eleva el consumo de cigarrillo $(3,4)$.

En Estados Unidos se ha calculado una prevalencia de 5,6\% en mayores de 25 años. Se dispone de poca información sobre la epidemiología de la EPOC en Colombia. La prevalencia de esta enfermedad y la magnitud de sus consecuencias varían ampliamente entre regiones, de ahí que la extrapolación de datos obtenidos en otros países no sea generalizable (3).

No obstante, el estudio PREPOCOL (Prevalencia de EPOC en Colombia) (2) muestra una prevalencia de $8,9 \%$ en mayores de 40 años (con un rango entre $6,2-13,5 \%$ ), lo que se traduce en una carga de enfermedad significativa para el país.

En 2005, la EPOC fue la tercera causa de años de vida sana perdidos (AVISA) para ambos sexos en personas de 45-59 años y la segunda causa en el grupo etario de 60 y más años $(2,5)$.

En el estudio del Ministerio de Salud "Carga de la enfermedad en Colombia” (2005) usando el mismo indicador, se estableció que la EPOC ocupa el séptimo lugar como causa de AVISA para todas las edades en el país (5).

Es, además, la cuarta causa de muerte en el mundo actual y se prevé un aumento de la mortalidad por esta enfermedad en las próximas décadas. En Colombia, fue la séptima causa de mortalidad en 2005 , con una tasa de 15,9 por 100.000 habitantes (5).

De otra parte, existen diversos tipos de fármacos disponibles que presuponen distintos mecanismos de acción. En los últimos años han surgido en el mercado combinaciones de dos fármacos a dosis fijas dentro de un mismo dispositivo. Por el momento, las combinaciones disponibles asocian un corticoide inhalado y un 32 -agonista de acción prolongada. Esta estrategia de tratamiento que en el asma ha supuesto 
beneficios clínicos importantes, se ha extendido a EPOC y hoy en día se cuenta con numerosos ensayos clínicos que han demostrado que el uso de un corticoide inhalado combinado con un 32 -agonista de acción prolongada en el mismo dispositivo de inhalación, es más efectivo que administrar alguno de sus componentes, incluso ciertos autores apuntan a la posibilidad de una mayor eficacia al dar los dos fármacos en un solo dispositivo frente a dar los dos fármacos en dos dispositivos distintos (6).

La mometasona/formoterol es un inhalador presurizado de dosis medidas (PDI) que contiene corticosteroides y un agonista beta de acción prolongada que proporciona un control eficiente de los síntomas en los casos de EPOC. La dosis recomendada es de dos inhalaciones, dos veces al día. El objetivo de esta investigación fue desarrollar un análisis del impacto presupuestal para los años 2013 a 2017 con la introducción de mometasona/ formoterol en el sistema de salud de Colombia. Este análisis estima las implicaciones financieras del uso de este medicamento en pacientes mayores de 40 años con EPOC (6).

\section{Materiales y métodos}

\section{Estructura del modelo}

El modelo requiere tres categorías principales de entrada de datos (figura 1):

- Población de pacientes (tamaño de la población, prevalencia de EPOC en Colombia, porcentaje tratado con combinaciones de dosis fijas-CDF-).

- Tratamientos de entrada (participación en el mercado, dosis prescritas).

- Costos promedio (de acuerdo con el SISMED 2013).

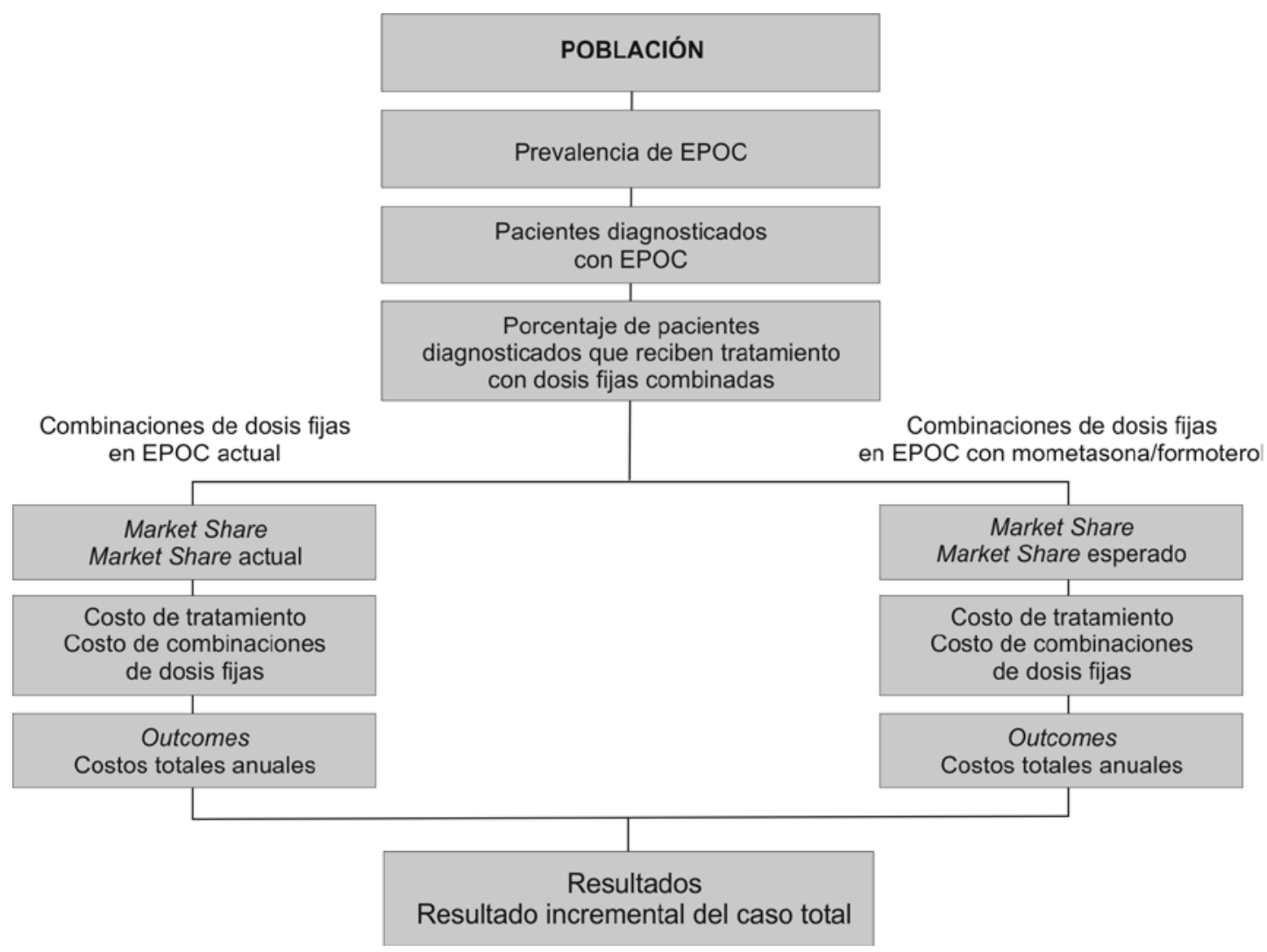

Figura 1. Estructura del modelo. 


\section{Población del modelo y perspectiva}

La población (caso base) está limitada a pacientes con 40 años de edad o más. La población estimada de acuerdo con los datos del DANE es 15.302.935 millones de colombianos/colombianas (7). La prevalencia ponderada de EPOC es de aproximadamente $8,9 \%$ considerando población mayor de 40 años (el estudio PREPOCOL (2) evaluó la prevalencia de EPOC en personas mayores de 40 años y reveló una prevalencia de $8,9 \%$ en cinco ciudades colombianas estudiadas). La información sobre el porcentaje de pacientes con EPOC en quienes se realizó un diagnóstico de la enfermedad, se extrajo de la literatura $(3,8)$; se hizo una estimación de los pacientes que usan CDF. La perspectiva es la del sistema de salud colombiano (tabla 1).

\section{Participación del mercado y costos de las combi- naciones de dosis fijas}

La participación en el mercado para las CDF se prevé según estimaciones de la unidad de negocios de cuidado primario y Market Access en MSD Colombia basados en la venta de unidades reportada al SISMED en el canal institucional durante el año 2013 y en la información reportada por la $I M S$ Health. Los costos de las CDF para el modelo del caso base se presentan según los precios mínimos y máximos reportados al SISMED 2013 en pesos colombianos. El precio total por dosis se determina dividiendo el costo por paquete por el número de dosis en un solo paquete. El costo anual total para un paciente se determina multiplicando el costo por dosis por el número de dosis indicadas durante un año.

Tabla 1. Población Modelo basado en la población.

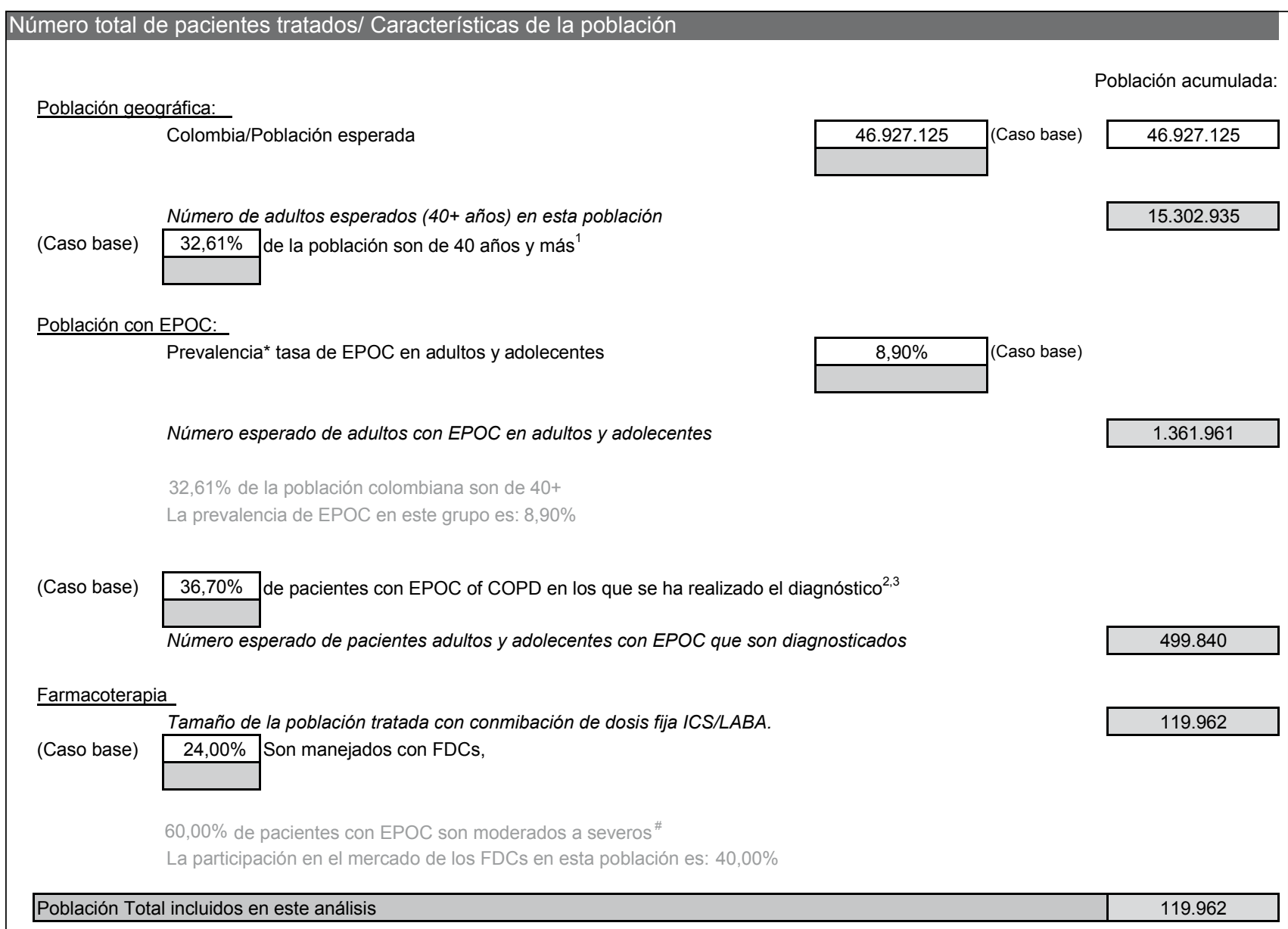




\section{Resultados}

\section{Caso base}

Los costos netos anuales (2013-2017) con la inclusión de mometasona/formoterol fueron $\$ 88.052$; \$88.052; \$88.053; \$88.053 y \$88.054 millones de pesos colombianos respectivamente. El impacto del presupuesto total de la utilización de mometasona/formoterol (sin descuento) en un período de tiempo de cinco años es de $\$ 11$ (once millones de pesos colombianos aproximadamente).

\section{Análisis de sensibilidad}

Este se enfocó sobre el impacto total en el presupuesto y su relación con la adherencia de las CDF (figura 2).

\section{Discusión}

El impacto del presupuesto total de la utilización de mometasona/formoterol (sin descuento) en un período de tiempo de cinco años es de 11 millones de pesos colombianos aproximadamente, con lo cual se tendría un impacto neutro sobre el presupuesto, pues el gasto actual es de $\$ 88.050$ millones de pesos y con inclusión en el año uno sólo aumenta a $\$ 88.052 \mathrm{mi}-$ llones de pesos y al final de los cinco años $\$ 88.054$ millones de pesos.
La mometasona/formoterol) es una combinación de dosis fija (inhalador de dosis medida presurizado que contiene un corticoide inhalado y un agonista $\beta 2$-adrenérgico de acción prolongada) que proporciona un control eficaz de los síntomas de EPOC en los pacientes mayores de 12 años tratados previamente con dosis media o alta de corticosteroides inhalados. La dosis recomendada es de dos inhalaciones dos veces al día.

El modelo presentado estimó las consecuencias financieras de la utilización de este medicamento en pacientes con EPOC mayores de 40 años de edad. Se utilizó esta población en el modelo dado que se trata del grupo poblacional con mayor prevalencia de EPOC y del cual se dispone de información epidemiológica (2).

El análisis de impacto presupuestal (AIP), o budget impact analysis, su equivalente en inglés, es parte esencial de la evaluación de tecnologías sanitarias y se utiliza cada vez más, junto con la evaluación económica, como un requisito para la inclusión de tecnologías o medicamentos en los planes de servicios de los sistemas de salud. El objetivo primordial es establecer si la nueva intervención o tecnología es pagable independientemente de su

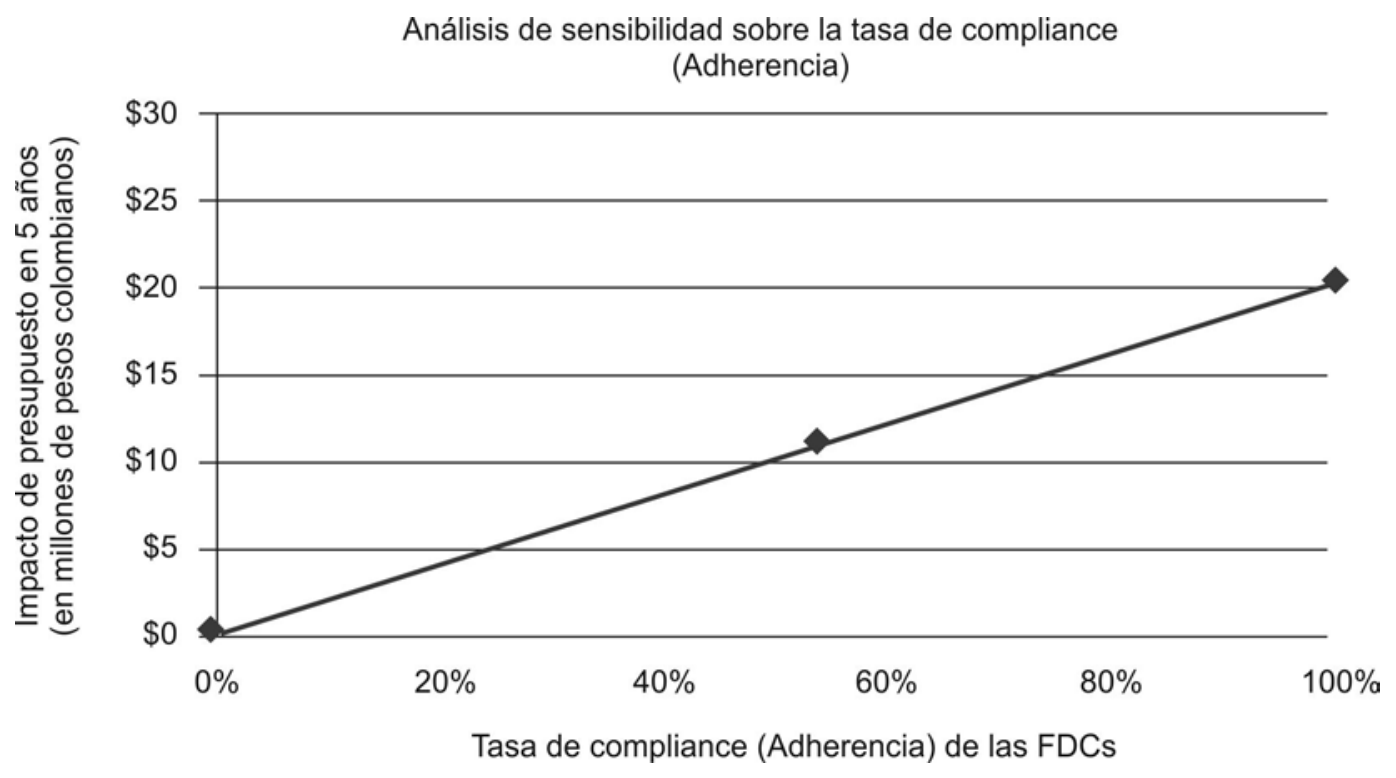

Figura 2. Análisis de sensibilidad univariado, adherencia a las combinaciones de dosis fijas. 


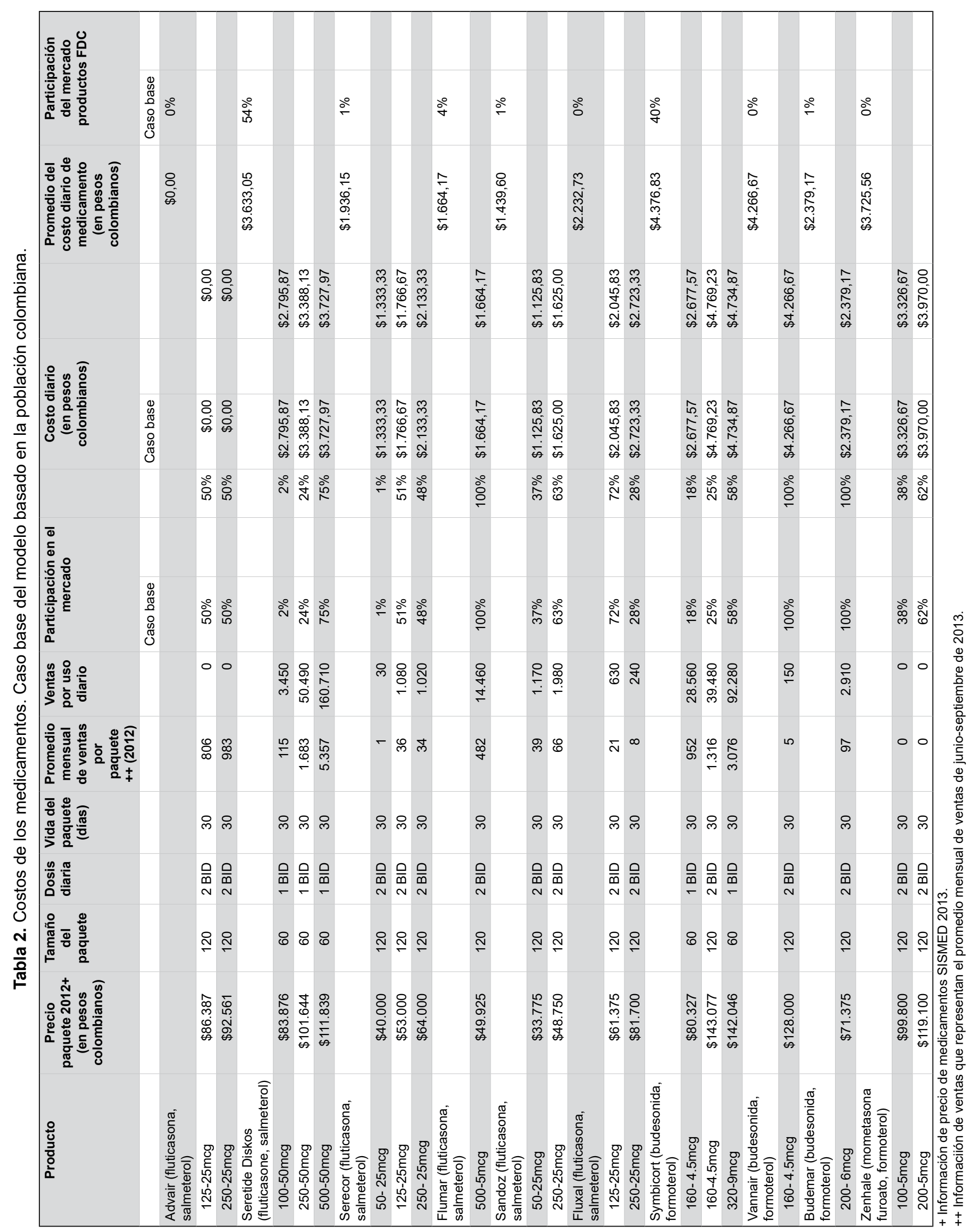


Tabla 3. 5-años de análisis de impacto.

\begin{tabular}{|c|c|c|c|c|c|c|c|c|c|c|c|}
\hline \multirow{2}{*}{$\begin{array}{l}\text { Costos } \\
\text { (en millones } \\
\text { de pesos } \\
\text { colombianos) }\end{array}$} & \multirow[t]{2}{*}{ Actual } & \multicolumn{5}{|c|}{$\begin{array}{l}\text { Costos total de medicamento proyectado } \\
\text { después de la entrada de Zenhale } \\
\text { sin descuento }\end{array}$} & \multicolumn{5}{|c|}{$\begin{array}{c}\text { Costos total de medicamento proyectado } \\
\text { después de la entrada de Zenhale } \\
\text { con descuento }\end{array}$} \\
\hline & & Año 1 & Año 2 & Año 3 & Año 4 & Año 5 & Año 1 & Año 2 & Año 3 & Año 4 & Año 5 \\
\hline $\begin{array}{l}\text { Costo total de } \\
\text { farmacia por } \\
\text { año }\end{array}$ & $\$ 88,050$ & $\$ 88,052$ & $\$ 88,052$ & $\$ 88,053$ & $\$ 88,053$ & $\$ 88,054$ & $\$ 88,052$ & $\$ 85,488$ & $\$ 82,998$ & $\$ 80,581$ & $\$ 78,234$ \\
\hline $\begin{array}{l}\text { Impacto de } \\
\text { presupuesto } \\
\text { de Zenhale }\end{array}$ & & $\$ 1,310$ & $\$ 2,040$ & $\$ 2,340$ & $\$ 2,690$ & $\$ 3,380$ & $\$ 1,310$ & $\$ 1,980$ & $\$ 2,210$ & $\$ 2,460$ & $\$ 3,000$ \\
\hline $\begin{array}{l}\text { Impacto del } \\
\text { presupuesto } \\
\text { total en } 5 \\
\text { años }\end{array}$ & & \multicolumn{5}{|c|}{$\$ 11,76$} & \multicolumn{5}{|c|}{$\$ 10,96$} \\
\hline
\end{tabular}

relación de costo-efectividad. La metodología del impacto de presupuesto presupone la medición del presupuesto antes de la inclusión de la nueva tecnología así como después de su inclusión en un periodo determinado (cinco años). La diferencia entre el valor inicial y el posterior constituye el impacto en el presupuesto (9).

Los resultados muestran como el presupuesto para las CDF antes del ingreso de la mometasona/ formoterol era de $\$ 88.050 .000$ y el impacto sobre el mismo que se genera una vez ingresa mometasona/ formoterol para el primer año es de $\$ 1.310 .000$, con un incremento en el presupuesto de menos del $0,5 \%$.

Cuando se evalúan los cinco años posteriores a la entrada de la mometasona/formoterol utilizando una tasa de descuento constante del 3\% (como lo recomiendan la guía metodológica para la atención de Guías de atención integral en el sistema de seguridad social colombiano del Ministerio de Protección Social, año 2010) (10), el costo total de las CDF se

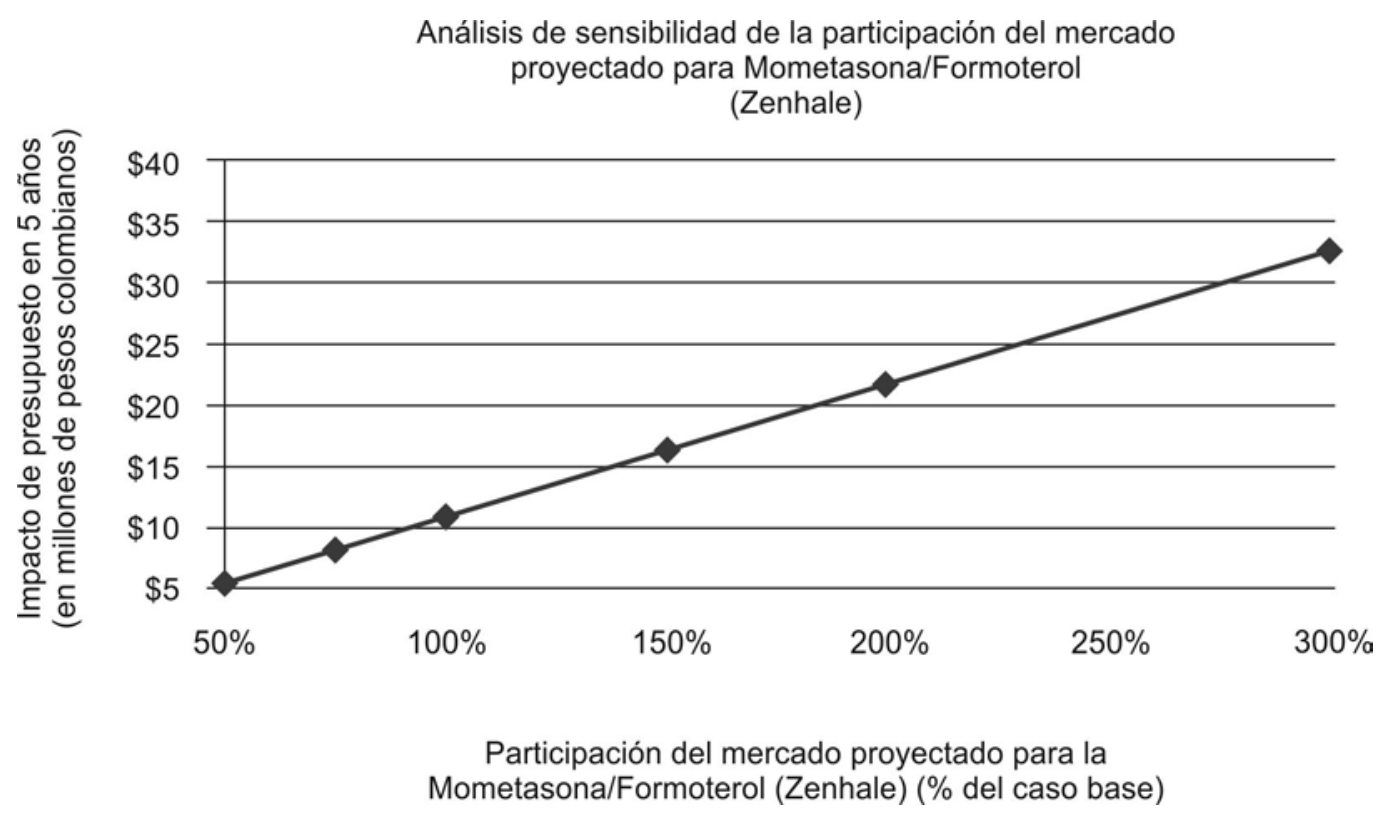

Figura 3. Análisis de sensibilidad univariado de la participación del mercado de la mometasona/formoterol. 
reduce año por año entre el 3 y el 12\%. Esta disminución (ahorro) en el costo de las CDF se explica por la sustitución parcial que genera el ingreso de la mometasona/formoterol y la disminución del uso de otras CDF (presentaciones de budesonida/formoterol así como de fluticasona/salmeterol).

Los resultados del análisis del impacto del presupuesto evidencian que la inclusión de mometasona/ formoterol en el tratamiento de la EPOC en comparación con otras $\mathrm{CDF}$, tienen el potencial de reducir los costos totales del presupuesto en el sistema de salud colombiano.

Así mismo, los resultados de este modelo de impacto económico, apoyan el ingreso de mometasona/formoterol al formulario POS del país, teniendo en cuenta que hoy no se cuenta con una combinación fija de esteroide inhalado y $\beta 2$ de acción prolongada en el formulario.

\section{Bibliografía}

1. Schiavi E, Stirbulov R, Hernandez Vecino R, Mercurio S, Di Boscio V, en nombre del Equipo Puma. COPD screening in primary care in four latinamerican countries: Methodology of the PUMA study. Arch Bronconeumol. 2014 May 7. pii: S0300-2896(14)00118-5. doi: 10.1016/j.arbres.2014.03.006.

2. Caballero A, Torres-Duque CA, Jaramillo C, Bolivar F, Sanabria F, Osorio P, et al. Prevalence of COPD in five colombian cities situated at low, medium, and high altitude (PREPOCOL study). Chest. 2008;133(2):343-9.
3. Cunningham TJ, Ford ES, Rolle IV, Wheaton AG, Croft JB. Associations of self-reported cigarette smoking with chronic obstructive pulmonary disease and co-morbid chronic conditions in the United States. COPD. 2015 Jun;12(3):276-86.

4. Chronic obstructive pulmonary disease (COPD) [Internet]; 2008. (Consultado 06 Jun 2013). Disponible en: http://www. who.int/mediacentre/factsheets/fs315/en/.

5. Acosta N, Peñaloza R, García J. Desarrollar el plan de análisis y la elaboración de informes del análisis de situación de salud de Colombia 2002 - 2007. 01/08/2005. Report No.: ASS/1502-08.

6. Dekhuijzen PN, Bjermer L, Lavorini F, Ninane V, Molimard M, Haughney J. Guidance on handheld inhalers in asthma and COPD guidelines. Respir Med. 2014;108(5):694-700.

7. Proyecciones Nacionales y Departamentales de población 2005-2020 [Internet]; 2014. [Consultado 06 Jun 2013]. Disponible en: http://www.dane.gov.co/.

8. Lindberg A, Bjerg A, Ronmark E, Larsson LG, Lundback B. Prevalence and underdiagnosis of COPD by disease severity and the attributable fraction of smoking report from the obstructive lung disease in northern sweden studies. Respir Med. 2006;100(2):264-72.

9. Sullivan SD, Mauskopf JA, Augustovski F, Jaime Caro J, Lee KM, Minchin M, et al. Budget impact analysis-principles of good practice: Report of the ISPOR 2012 budget impact analysis good practice II task force. Value Health. 2014;17(1):5-14.

10. Guía metodológica para la elaboración de guías de atención integral en el sistema general de seguridad social en salud colombiano. [Internet]; 2010. [Consultado 06 Jun 2013]. Disponible en: https:/www.minsalud.gov.co/salud/Documents/ Gu\%C3\%ADa\%20Metodo1\%C3\%B3gica\%20para\%20 la\%20elaboraci\%C3\%B3n\%20de $\% 20$ gu\%C3\%ADas.pdf 